\title{
Three-Dimensional Reconstruction Algorithm for CT Pulmonary Angiography in Patients with Pulmonary Embolism Combined with Syncope
}

\author{
Zhongxing Zhang $\mathbb{D}^{1},{ }^{1}$ Yan Zhang $\mathbb{D}^{2},{ }^{2}$ Xiaohui Wang ${ }^{\mathbb{D}},{ }^{1}$ Xiaoli Han ${ }^{\mathbb{D}},{ }^{1}$ Xin Zhang $\mathbb{D}^{1}$ \\ and Hong Chen (iD) \\ ${ }^{1}$ Department of Respiratory and Critical Care Medicine, The First Affiliated Hospital of Chongqing Medical University, \\ Chongqing 400010, China \\ ${ }^{2}$ Department of Radiology, Affiliated Hospital of Chongqing Three Gorges Medical College, Chongqing 400010, China
}

Correspondence should be addressed to Hong Chen; 3160200145@caa.edu.cn

Received 21 August 2021; Revised 8 October 2021; Accepted 11 October 2021; Published 2 December 2021

Academic Editor: Gustavo Ramirez

Copyright (c) 2021 Zhongxing Zhang et al. This is an open access article distributed under the Creative Commons Attribution License, which permits unrestricted use, distribution, and reproduction in any medium, provided the original work is properly cited.

\begin{abstract}
This paper aimed to study the clinical characteristics of patients with pulmonary embolism (PE) with syncope $(\mathrm{PE}+\mathrm{S})$ based on the three-dimensional (3D) reconstruction algorithm in computed tomography (CT) pulmonary angiography (CTPA). In this study, 857 patients with acute PE (APE) who were treated in hospital were selected as the research objects and divided into syncope group (group S) and nonsyncope group (group NS). The 3D reconstruction marching cubes (3DR-MC) algorithm was compared with the traditional MC (T-MC) algorithm and the mesh simplification MC (MMS-MC) algorithm, and the results proved that the running time of the 3DR-MC algorithm on the platform was shorter than that of the other two algorithms. The incidence of syncope in group S in women was higher than that in group NS (51.7\% vs. 38.2\%). The incidence of syncope classified as high risk in group $S$ was higher than that in group NS, and the mortality rate of pulmonary embolism patients with syncope was higher, and the difference was statistically significant $\left(\chi^{2}=113.332, P<0.05\right)$. The incidence of syncope in group $S$ was higher than that in group NS $\left(\chi^{2}=4.074, P<0.05\right)$. In short, hypertension was an independent risk factor for syncope. $\mathrm{PE}+\mathrm{S}$ patients could be diagnosed and treated as early as possible based on the clinical characteristics, so as to reduce the adverse consequences of misdiagnosis.
\end{abstract}

\section{Introduction}

APE refers to a pathological syndrome in which endogenous or exogenous emboli block the main trunk or branches of the pulmonary artery, leading to pulmonary circulatory disorders. Its mortality rate is the second only to tumors and myocardial infarction [1]. Clinically, there are many factors that cause syncope in patients, and the heart and cerebrovascular diseases are considered firstly. However, syncope caused by PE is often ignored, which increases the false negative rate (FNR) and false positive rate (FPR). Previous studies have shown that syncope is one of the common symptoms of PE and $17.3 \%$ of PE patients taking syncope as the first symptom [2]. Sivilotti et al. [3] pointed out that the incidence of $\mathrm{PE}+\mathrm{S}$ was about $22 \%$. Therefore, $\mathrm{PE}+\mathrm{S}$ deserves the clinical attention to analyze the clinical characteristics of its patients, thereby reducing the FNR and FPR.

CT uses X-rays to scan a certain part of the human body together with a highly sensitive detector. It has the characteristics of fast scanning time and clear scanned images [4]. The imaging principle is to use energy rays to scan an object, rely on projection data obtained from the outside of the object, and use a specific reconstruction algorithm to present the tomographic imaging of the inside of the object in the form of a $2 \mathrm{D}$ or 3D image. In recent years, CT images have been widely used in the field of medical clinical 
diagnosis. Tomography is performed on the injured parts of the human body, and the disease is determined by analyzing the images. CT examination has become an indispensable way in the medical field of lung diagnosis [5]. With the continuous update of the $3 \mathrm{D}$ cone-beam reconstruction algorithm, the MC algorithm proposed by Chacón et al. [6] is relatively prominent, although it can only get good results when the reconstruction cone angle is small. However, it has the advantages of simple operation, effective method, and fast effect, so the MC algorithm has always been the main algorithm used in actual projects.

The innovation of this study is based on the 3DR-MC algorithm, which is compared with the traditional MC (TMC) algorithm and mesh simplification (S-MC) algorithm and applied to CT pulmonary angiography of 857 patients with pulmonary embolism. The objective of this study was to investigate the differences in clinical characteristics and mortality during hospitalization in PE patients with and without syncope, so as to provide reliable reference and assistance for clinical diagnosis.

\section{Materials and Methods}

2.1. General Information. 857 patients who were medically diagnosed as APE patients in the hospital from January 2015 to January 2017 were selected as the research objects in this study, including 522 males and 335 females. They were divided into group S (58 cases, $65.1 \pm 14.8$ years old) and group NS (799 cases, $61.6 \pm 15.8$ years old) according to whether they were accompanied by syncope. The study had been approved by the Medical Ethics Committee of Hospital, and the patients and their families had understood the study and signed the informed consents.

Inclusion criteria were defined as follows: APE patients diagnosed by CTPA examination with available imaging data; patients with acute onset; patients with detailed hospitalization records in this hospital; and patients without thrombolytic therapy before admission.

The exclusion criteria were determined as follows: patients with chronic PE who were repeatedly hospitalized; patients with only clinical diagnosis and lack of direct evidence; patients who were suggested with embolism by CTPA and other examinations but not clinically consistent; patients with coronary heart disease; and patients with unclear CT images.

2.2. Research Methods. The medical history of the two groups of patients was collected, including the history of previous diseases (cardiovascular disease (CVD), respiratory disease, venous thrombus embolism (VTE), and diabetes), symptoms at the onset (chest pain, cough, hemoptysis, dyspnea, etc.), and signs of syncope (shock, accelerated heart rate, and electrocardiograph (ECG) changes), biochemical examination (brain natriuretic peptide (BNP) and troponin), echocardiography (right ventricular dysfunction (RVD)), venous thrombosis of the lower limbs or not, CTPA examination (a large area thrombus or not), PE severity index (PESI), and PE-related mortality.
The collected clinical indicators were based on the data from the first examination after the onset of syncope symptoms to the doctor within 24 hours.

2.3. Diagnostic Criteria. The diagnostic criteria of syncope referred to the 2009 European Society of Cardiology (ESC) guidelines, which were sudden and short loss of consciousness, which was featured with fast onset, short duration, and self-limiting [7]. The PESI was determined based on the recommendations of the 2014 ESCAPE guidelines:

(1) The low-risk group referred to the absence of RVD and shock, and the score was 0 .

(2) The moderately low-risk group referred to the combination of RVD or elevated myocardial injury markers, and PESI score was no lower than 1.

(3) The moderately high-risk group referred to the presence of both RVD and elevated myocardial injury markers, and the PESI score was no lower than 1.

(4) The high-risk group referred to shock, RVD, and increased myocardial injury markers, and the PESI score was no lower than 1.

The PESI scoring criteria was to add 1 score to PE patients for one the following items, including tumor, chronic heart failure or chronic lung disease, heart rate $\geq 110$ beats/min, systolic blood pressure (BP) $<100 \mathrm{mmHg}$, and oxygen saturation $<90 \%$ [8]. The diagnostic criteria of myocardial injury referred to the standards proposed by the American Heart Association (AHA) in 2011, and it was diagnosed when the myocardial injury marker cardiac troponin I (cTnI) $>0.4 \mathrm{ng} /$ $\mathrm{ml}$ in PE patients [9]. RVD was defined as an echocardiographic examination showing right ventricular dilation (end diastolic right ventricular diameter/left ventricular diameter $>0.9$ or 1.0). Shock was defined as systolic BP less than $90 \mathrm{mmHg}$, average arterial pressure less than $65 \mathrm{mmHg}$, or a decrease of $\geq 40 \mathrm{mmHg}$ from the baseline [10].

2.4. CT Examination. The dual source CT scanner (SOMATOM Definition, Siemens Medical Solutions, Forchheim, Germany) was adopted. Before the examination, the patient was instructed to remove the metal jewelry on the chest, tested with an iodine allergy in advance, explained the scanning process, and required to maintain a calm mind. Firstly, the scan range was from the thoracic entrance to the diaphragmatic level. Then, an enhanced scan was taken. The contrast agent was injected using the bolus injection method. The injection rate of contrast agent iopromide injection was $4 \mathrm{~mL} / \mathrm{s}$, and the injection volume was $40 \mathrm{~mL}$. Then, $30 \mathrm{~mL}$ of normal saline (NS) was injected in the same way to reduce the concentration of contrast agent in the superior vena cava. And, the scanning time was delayed for 3 seconds. Care dose $4 \mathrm{D}$ and care $\mathrm{kV}$ modes were used for scanning, the scanning layer thickness was $0.625 \mathrm{~mm}$, the scanning interval was $0.625 \mathrm{~mm}$, the pitch was $1.2: 1$, the matrix was $512 \times 512$, the voltage was $100 \mathrm{kV}$, and the current was $200 \mathrm{~mA}$. 
2.5. Calculating Steps of 3DR-MC Algorithm. The basic research unit of the MC algorithm was a voxel, which was a cube structure composed of 8 vertices in adjacent parts of the data field (as shown in Figure 1).

Firstly, the position of each edge in the voxel was determined, and then the cross coordinates of the intersection point was determined. It meant that determining the positions of all the vertices of the triangle surface can determine the location coordinates of the triangle vertices in the voxel in space. The traditional algorithm adopted the linear interpolation to calculate. It was assumed that the edge of each side of the voxel was parallel to the axis $a, b$, and $c$. The vertex coordinates $S(a, b, c)$ of the triangular surface were defined as follows:

$$
\begin{aligned}
& a=a_{1}+\frac{\left(F-h_{1}\right) \cdot\left(a_{2}-a_{1}\right)}{h_{2}-h_{1}}, \\
& b=b_{1}+\frac{\left(F-h_{1}\right) \cdot\left(b_{2}-b_{1}\right)}{h_{2}-h_{1}}, \\
& c=c_{1}+\frac{\left(F-h_{1}\right) \cdot\left(c_{2}-c_{1}\right)}{h_{2}-h_{1}} .
\end{aligned}
$$

In the above equations, $S_{1}\left(a_{1}, b_{1}, c_{1}\right)$ and $S_{2}\left(a_{2}, b_{2}, c_{2}\right)$ were the coordinates of the vertices of the two edges of the voxel, and $h_{1}$ and $h_{2}$ were the gray values of the two vertices in $S_{1}$ and $S_{2}$; and $F$ referred to the determined threshold. In order to make the reconstructed function image clearer, it was necessary to calculate the normal vector of the triangle surface, use the central difference method to calculate the gradient value of the vertices in the voxel, and then solve the triangle surface vertex normal vector with linear interpolation. The gradient equations for vertex coordinates of the voxel edge could be expressed as follows:

$$
\begin{aligned}
& H_{a}=\frac{M_{(a+p, b, c)}-M_{(a-p, b, c)}}{2 p}, \\
& H_{b}=\frac{M_{(a, b+q, c)}-M_{(a, b-q, c)}}{2 q}, \\
& H_{c}=\frac{M_{(a, b, c+s)}-M_{(a, b, c-s)}}{2 s} .
\end{aligned}
$$

In the above equations, $p, q$, and $s$ represented the side length of the cube, $M(a, b, c)$ indicated the function value of the coordinate point $(a, b, c)$, referring to the gray value; and $H_{a}, H_{b}$, and $H_{c}$ represented the gradient values in the three directions. To obtain the gradient value of the voxel edge length vertex, the normal vector $R\left(R_{a}, R_{b}, R_{c}\right)$ of the triangle vertices had to be available. The specific equation could be written as follows:

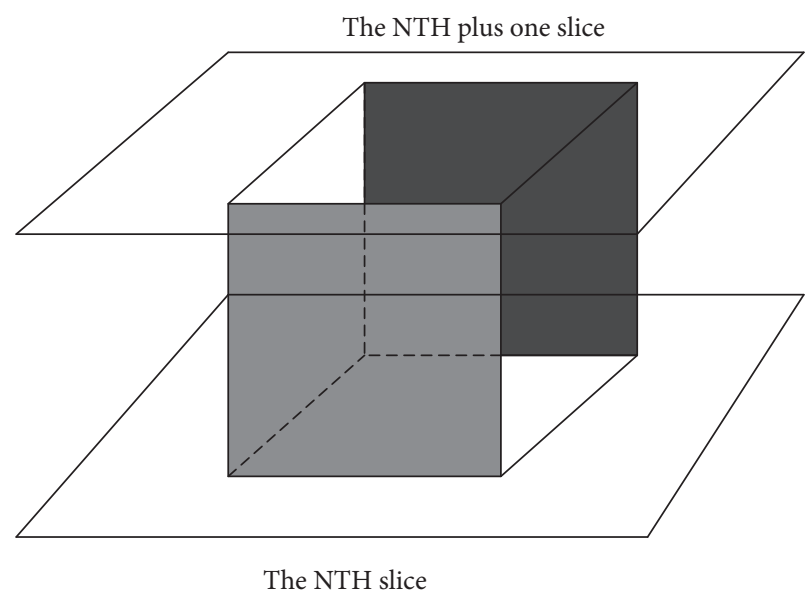

FIgURE 1: Voxel of the basic research unit.

$$
\begin{aligned}
& R_{a}=R_{a 1}+\frac{\left(F-h_{1}\right) \cdot\left(R_{a 2}-R_{a 1}\right)}{\Delta h}, \\
& R_{b}=R_{b 1}+\frac{\left(F-h_{1}\right) \cdot\left(R_{b 2}-R_{b 1}\right)}{\Delta h}, \\
& R_{c}=R_{c 1}+\frac{\left(F-h_{1}\right) \cdot\left(R_{c 2}-R_{c 1}\right)}{\Delta h} .
\end{aligned}
$$

In the above three equations, $R\left(R_{a 1}, R_{b 1}, R_{c 1}\right)$ and $R\left(R_{a 2}\right.$, $R_{b 2}, R_{c 2}$ ) were the normal vectors at the two vertices of the edge of the voxel; and $F$ referred to the determined threshold. $\Delta h=h_{2}-h_{1}$, where $H_{2}$ and $H_{1}$ were the gray values of the vertex of the edge. The appropriate voxels of equal face value were selected to calculate the normal vector and the equivalent point. In this study, the golden section point of the edge was replaced to the linear interpolation, and the related equations were as follows:

If the cross of edges was located at axis $a, b$, and $c$, the cross coordination could be calculated with $(a+(\sqrt{5-1}) / 2, b, c),(a, b+(\sqrt{5}-1) / 2, c)$, and $(a, b, c+$ $(\sqrt{5}-1) / 2)$, respectively.

If the cross of edges was located at axis $a, b$, and $c$, the normal vector of the cross could be calculated with $R=$ $R(a, b, c)+((\sqrt{5}-1) / 2)(R(a+1, b, c)-R(a, b, c)), \quad R=$ $R(a, b, c)+((\sqrt{5-1}) / 2)(R(a, b+1, c)-R(a, b, c))$, and $R=R(a, b, c)+((\sqrt{5-1}) / 2)(R(a, b, c+1)-R(a, b, c))$, respectively.

In the above equations, $R=R(a, b, c)$ referred to the vector of coordination $(a, b, c)$ and $R$ represented the normal vector.

2.6. Statistical Methods. The SPSS 22.0 statistical software was employed for analysis. The Kolmogorov-Smirnov method was to test the normality of continuous variables. The data met the normal distribution were represented by the mean \pm standard deviation. The independent sample $t$ test was applied for comparison between groups. The data of nonnormal distribution was expressed with median (P25, P75). The comparison was taken using the Mann-Whitney $U$ rank sum test. The categorical variables were verified with 
Pearson 2 and Fisher, and $P<0.05$ indicated statistical difference. Multivariate unconditional logistic regression analysis was performed on variables with statistical meaningfulness in univariate analysis.

\section{Results}

3.1. Comparison on Reconstruction Effects of Three Algorithms. The 3DR-MC algorithm constructed in this study was compared with the T-MC algorithm and the MS-MC algorithm in reconstructing pulmonary lobe, and the results are illustrated in Figure 2. The running time of the proposed algorithm was shorter than that of the T-MC and MS-MC algorithms with obvious difference $(P<0.05)$. The results showed that the proposed algorithm was adjusted on the basis of the traditional algorithm, which could reduce the reconstruction time under the premise of ensuring the reconstruction effect, thereby effectively satisfying the needs of medical research.

3.2. General Information of Two Groups of Patients. There was no observable difference in age between the two groups of patients (Figure 3). In addition, there was no huge difference in the comparison of comorbidities such as gender, hypertension, and diabetes, which can be studied and compared.

\subsection{CT Imaging Characteristics of PE Patients. During the} CT scan, high-density or low-density foci could be seen on the main pulmonary artery and the main trunks or branches of the left and right pulmonary arteries. The high-density foci represented fresh thrombus, and the low-density foci represented old thrombus. Pulmonary artery obstruction signs could be observed, such as regional lung texture thinning, sparseness or disappearance, and increased lung field transparency. In addition, it could be manifested as widening of the right lower pulmonary artery or accompanied by truncation signs, pulmonary artery segment bulging, right ventricular enlargement, and other signs of pulmonary hypertension. PE caused pulmonary arteries to change, which was manifested as local triangular lung tissue incidents. The consolidated lung tissue may become necrosis, forming a cavity. Short-term changes in the cavity were great, deep vein thrombosis was formed in the lower limbs, and pulmonary artery cavity suffered from filling defect (as shown in Figure 4).

During the enhanced CT scan, the embolized pulmonary arteries were dilated and thickened, and there were some long strips or irregular filling defects in the embolized part, showing low-density (as given in Figure 5). Some patients showed partial shadows in the lung field, wedge-shaped shadows with the tip pointing to the hilum, atelectasis and insufficiency, and other secondary changes in lung tissue. On the side with atelectasis, the diaphragm could be elevated and combined with less to mid amount of pleural effusion sometimes.

\subsection{Clinical Characteristics of Patients in Group S and Group} NS. The incidence of syncope among $857 \mathrm{PE}$ patients was $6.8 \%$. The incidence of group $\mathrm{S}$ in women was higher than

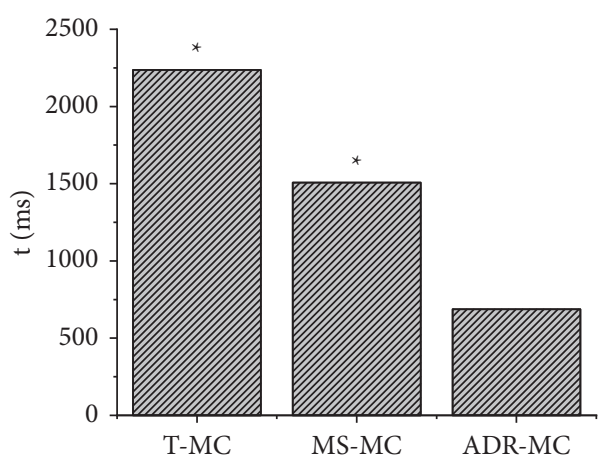

FIgURE 2: Comparison of running time of three algorithm platforms. Note: ${ }^{*}$ indicated $P<0.05$ in contrast to 3DR-MC.

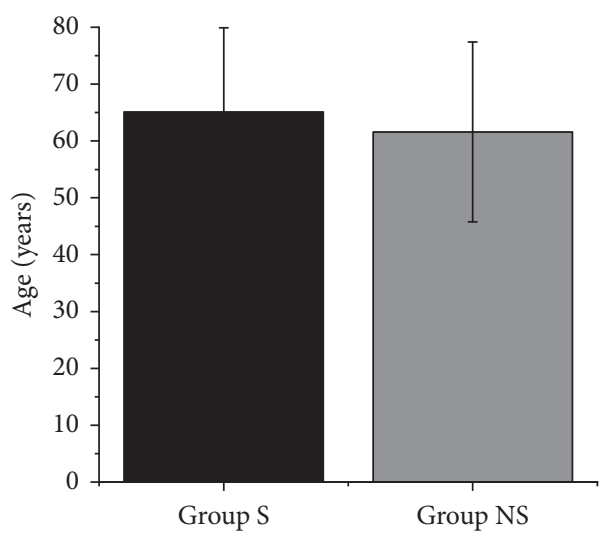

FIGURE 3: Comparison on ages of two groups of patients.

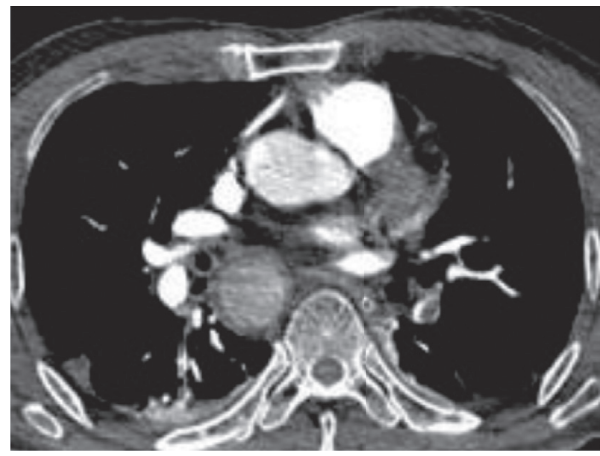

FIgURE 4: Consolidation of ischemic area, pulmonary artery filling defect.

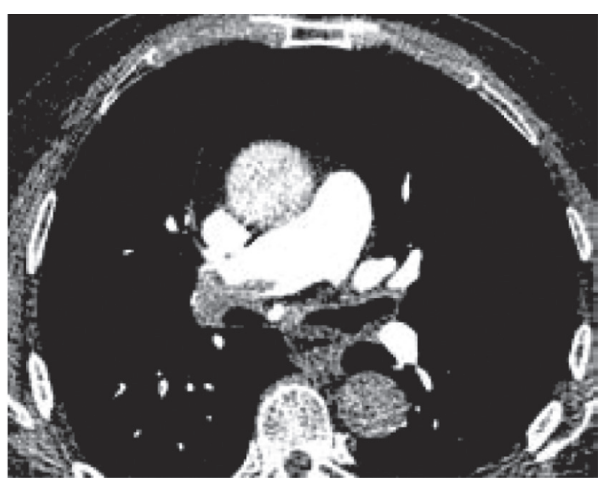

FIGURE 5: Dilated and thickened pulmonary artery. 
TABle 1: Clinical characteristics of patients in group S and group NS.

\begin{tabular}{|c|c|c|c|c|}
\hline Variable (cases, proportion) & Group S $n=58(\%)$ & Group NS $n=799(\%)$ & $\chi^{2}$ & $P$ \\
\hline Male & $28(48.3)$ & $494(61.8)$ & 4.171 & 0.041 \\
\hline Female & $30(51.7)$ & $305(38.2)$ & 4.171 & 0.041 \\
\hline Age (years) & $65.1 \pm 14.8$ & $61.6 \pm 15.8$ & 0.039 & 0.841 \\
\hline Combined with basic CVD & $28(48.3)$ & $266(33.3)$ & 5.387 & 0.02 \\
\hline Hypertension & $25(43.1)$ & $224(28.0)$ & 5.956 & 0.015 \\
\hline Other CVD & $3(5.2)$ & $42(5.3)$ & 0.005 & 0.943 \\
\hline Combined with basic pulmonary diseases & $28(48.3)$ & $343(42.9)$ & 0.630 & 0.427 \\
\hline VTE history & $8(13.8)$ & $65(8.1)$ & 2.221 & 0.136 \\
\hline Diabetes & $10(17.2)$ & $102(12.8)$ & 0.953 & 0.329 \\
\hline Chest pain & $6(10.3)$ & $108(13.5)$ & 0.472 & 0.492 \\
\hline Cough & $14(24.1)$ & $228(28.5)$ & 0.516 & 0.473 \\
\hline Hemoptysis & $7(12.1)$ & $77(9.6)$ & 0.362 & 0.548 \\
\hline Breathing difficulties & $36(62.1)$ & $296(37)$ & 14.267 & $\leq 0.001$ \\
\hline $\mathrm{BP}$ at shock & $17(29.3)$ & $20(2.5)$ & 94.068 & $\leq 0.001$ \\
\hline \multicolumn{5}{|l|}{$E C G$} \\
\hline $\mathrm{RBBB}$ & $9(15.5)$ & $67(8.4)$ & 3.403 & 0.065 \\
\hline $\mathrm{S}_{\mathrm{I}} \mathrm{Q}_{\text {III }} \mathrm{T}_{\text {III }}$ & $16(27.6)$ & $75(9.4)$ & 18.871 & $\leq 0.001$ \\
\hline Increased heart rate & $31(53.4)$ & $270(33.8)$ & 9.169 & 0.002 \\
\hline \multicolumn{5}{|l|}{ Ultrasonic cardiogram } \\
\hline RVD & $21(36.2)$ & $69(8.6)$ & 42.075 & $\leq 0.001$ \\
\hline Increased BNP & $39(67.2)$ & $240(30)$ & 34.088 & $\leq 0.001$ \\
\hline Increased CTnI & $36(62.1)$ & $160(20)$ & 54.188 & $\leq 0.001$ \\
\hline Thrombolysis & $7(12.1)$ & $51(6.4)$ & 2.552 & 0.110 \\
\hline \multicolumn{5}{|l|}{ CTPA examination } \\
\hline Extensive thrombosis & $12(20.7)$ & $32(4)$ & 30.906 & $\leq 0.001$ \\
\hline Death in hospital & $10(17.2)$ & $22(2.8)$ & 31.577 & $\leq 0.001$ \\
\hline
\end{tabular}

Note. The rate $>100$ beats/min could be determined as increased heart rate. BP at shock was defined as the mean arterial pressure was less than $65 \mathrm{mmHg}$, the systolic BP was less than $90 \mathrm{mmHg}$, or $40 \mathrm{mmHg}$ lower than the basic value.

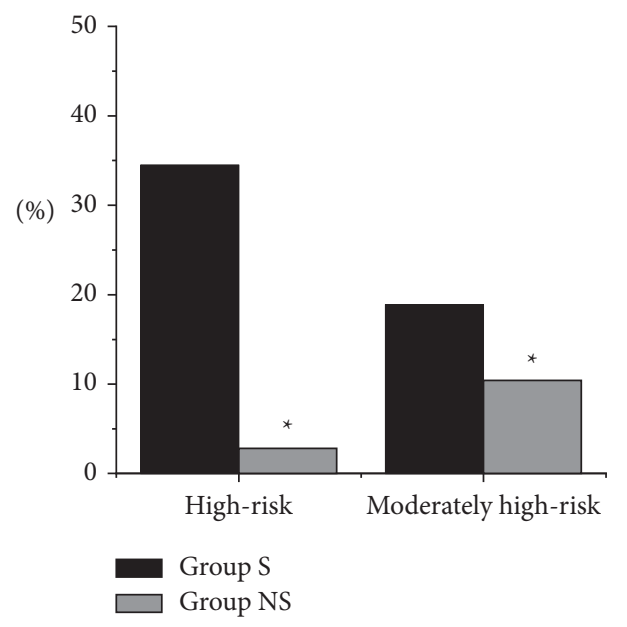

Figure 6: Incidences of high-risk and moderately high-risk in both groups. Note: ${ }^{*}$ indicated $P<0.05$ in contrast to group $\mathrm{S}$.

that in group NS (51.7\% VS. 38.2\%), dyspnea, BP at shock, increased troponin and BNP, $\mathrm{S}_{\mathrm{I}} \mathrm{Q}_{\mathrm{III}} \mathrm{T}_{\mathrm{III}}$ for $\mathrm{ECG}$, increased heart rate, increased CTnI, RVD, and extensive thrombosis. The difference was statistically obvious $(P<0.05)$ (Table 1$)$.

3.5. Risk Classification of Patients in Group S and Group NS. The incidences of high-risk and moderately high-risk in group $\mathrm{S}$ were higher, so the mortality of $\mathrm{PE}+\mathrm{S}$ patients was higher hugely in contrast to group NS $\left(\chi^{2}=113.332\right.$ and

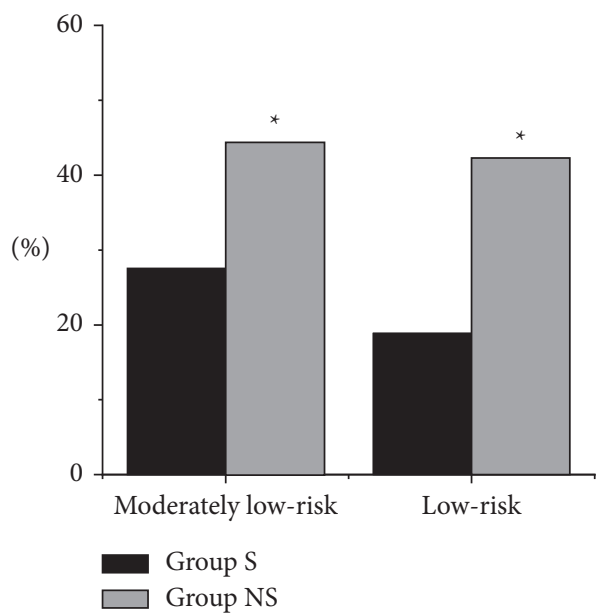

Figure 7: Incidences of low-risk and moderately low-risk in both groups. Note: ${ }^{*}$ indicated $P<0.05$ in contrast to group $S$.

$\chi^{2}=4.074$, respectively, $P<0.05$ ), which could be observed in Figure 6. Figure 7 illustrated that the incidences of moderately low-risk and low-risk in group $\mathrm{S}$ were much lower in contrast to group NS $\left(\chi^{2}=6.250\right.$ and $\chi^{2}=5.479$, respectively, $P<0.05)$.

3.6. Univariate Analysis of $P E+S$. The values to variables with obvious differences in univariate analysis were assigned: 1 for yes and 0 for no (taking gender, history of 
TABLE 2: Classifications and assignments of factors.

\begin{tabular}{lc}
\hline Variable & Assignment \\
\hline Gender & 0 for male and 1 for female \\
Hypertension & 0 for no and 1 for yes \\
Breathing difficulty & 0 for no and 1 for yes \\
BP at shock & 0 for no and 1 for yes \\
cTnI & 0 for no and 1 for yes \\
BNP & 0 for no and 1 for yes \\
$\mathrm{S}_{\mathrm{I}} \mathrm{Q}_{\mathrm{III}} \mathrm{T}_{\mathrm{III}}$ & 0 for no and 1 for yes \\
Increased heart rate & 0 for no and 1 for yes \\
$\mathrm{RVD}$ & 0 for no and 1 for yes \\
Extensive thrombosis & 0 for no and 1 for yes \\
\hline
\end{tabular}

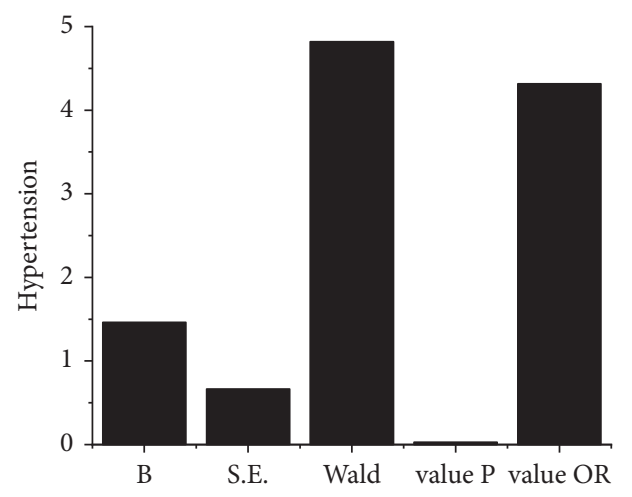

FIGURE 8: Incidence of syncope for PE patients with hypertension.

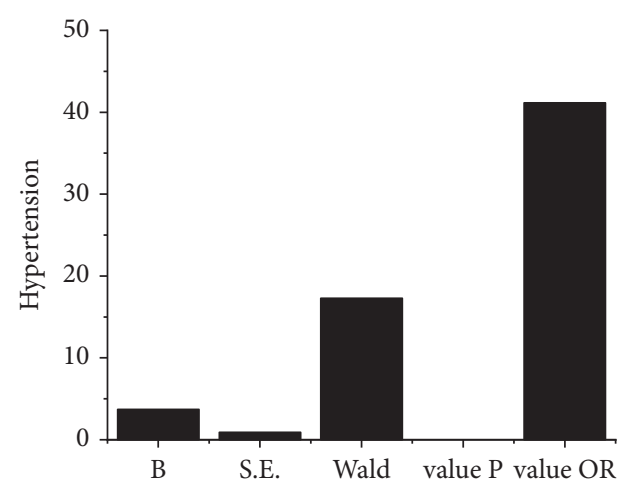

FIGURE 9: Incidence of syncope for PE patients with BP at shock.

hypertension, dyspnea, BP at shock, troponin and BNP, $\mathrm{S}_{\mathrm{I}} \mathrm{Q}_{\mathrm{III}} \mathrm{T}_{\mathrm{II}}$ on ECG, increased heart rate, $\mathrm{RVD}$, and extensive thrombosis as independent variables). The specific assignments are shown in Table 2.

3.7. Multivariate Analysis of $P E+S$. Multivariate unconditional logistic regression analysis found that hypertension, BP at shock, and extensive thrombosis were independent risk factors for syncope. PE patients with a history of hypertension were 4.3 times more likely to have syncope than those without hypertension (as illustrated in Figure 8). Figure 9 disclosed that $\mathrm{PE}$ patients with $\mathrm{BP}$ at shock were 41.1 times more likely to have syncope than those without BP at shock. PE patients with extensive thrombosis were 14.2

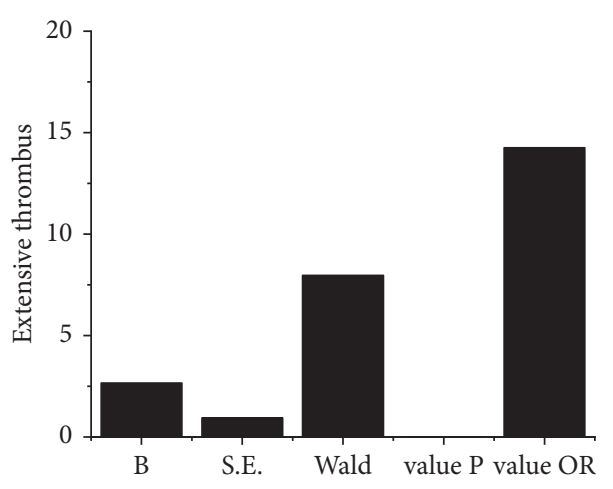

FIGURE 10: Incidence of syncope for PE patients with extensive thrombus.

times more likely to have syncope than those without it, and the specific values could be obtained in Figure 10.

\section{Discussion}

APE is a common clinical emergency and critical illness. The dyspnea and chest pain are the most common. The incidence of syncope in $\mathrm{PE}$ is much lower than that of dyspnea or chest pain [11]. Epstein et al. [12] pointed out that the incidence of syncope in patients with PE is about $22 \%$. In this study, the incidence of syncope was about $7 \%$, while the incidence of dyspnea and chest pain were about $41 \%$ and $14 \%$, respectively, which were consistent with previous studies. In the past, if a patient suffered from syncope, cardiogenic diseases or CVD were often given priority. Therefore, syncope caused by $\mathrm{PE}$ was misdiagnosed as a cardiogenic disease or CVD, which delayed the optimal treatment time for such patients and resulted in increased mortality. On the other hand, syncope is often a sign of fatal PE. If clinicians can accurately and quickly identify the cause of syncope and give appropriate treatment, mortality can be reduced greatly. Monté et al. [13] applied the traditional MC algorithm to CT images of 113 patients with $\mathrm{PE}$ and found that the reconstruction time of the traditional MC algorithm was longer. Sink et al. [14] simplified the running time of $\mathrm{MC}$ algorithm in CT examination based on the grid, and the reconstruction effect was relatively ideal. Based on the above research, the $3 \mathrm{DR}-$ $\mathrm{MC}$ algorithm was constructed in this study, which not only guaranteed the effect of CT image reconstruction in patients with PE but also greatly reduced the running time of the algorithm.

Comparing the A-MC algorithm constructed in this study with T-MC algorithm and S-MC algorithm in the reconstruction of left and right lung lobes, it can be found that the algorithm in this study has shorter running time on the platform than T-MC and S-MC algorithm, and the difference is statistically significant $(P<0.05)$. The A-MC algorithm can be adjusted on the basis of the traditional algorithm, which can reduce the reconstruction time and effectively meet the needs of medical research on the premise of ensuring the reconstruction effect. The occurrence of $\mathrm{PE}+\mathrm{S}$ is the result of multifactor interaction. It was found in this study that patients with hypertension, BP at shock, and 
extensive thrombosis were more likely to have syncope, and these three were independent risk factors for syncope. In this study, the incidence of hypertension in group $\mathrm{S}$ was relatively high (about $43 \%$ ). Syncope caused by hypertension may be due to a decrease in blood return after cerebral arteriosclerosis, causing transient cerebral ischemia. Previous studies have found that the risk of hypotension is higher especially in the early stages of syncope. In this study, the incidence of BP at shock during syncope was about $29 \%$, which may be due to the drop in BP due to PE, leading to arrhythmia. Compared with shock, patients with syncope are more likely to have RVD. Changes in RVD play a key role in the overall pathophysiological changes. Syncope is related to malignant arrhythmia, APE can also cause Arrhythmia, and RVD can increase the risk of malignant arrhythmia, leading to the occurrence of syncope. Previous studies have also shown that PE patients with syncope are more prone to RVD [15], which was consistent with this study. There were around $21 \%$ of patients with syncope suffered from extensive PE among the included objects. Previous studies have found that the incidence is about $13 \%-60 \%$ [16]. The probability of syncope in patients with extensive thrombosis was 14.2 times that of no extensive thrombosis, which may be caused by increased pulmonary hypertension and RVD after extensive PE and decreased left ventricular filling, leading to a decrease in cardiac output. Clinically, patients with extensive PE should be alert to syncope.

Since syncope in PE patients may indicate severe pathophysiological changes, its prognosis has always been the focus of attention. Many studies have shown that the PESI score is a highly reliable clinical prognostic indicator for PE patients. The higher the PESI score, the higher the mortality rate of PE patients. In this study, the proportions in group $\mathrm{S}$ classified as high-risk and moderately high-risk were greater than those of group NS, and the mortality rate in group S was also higher, reaching $17.2 \%$, which was consistent with previous studies [17]. Sulaiman et al. [18] found that a previous history of syncope is one of the independent risk factors for increased mortality during hospitalization, especially in elderly patients. Autopsy studies on PE patients also confirmed that the prominent symptoms in PE patients included the sudden syncope.

\section{Conclusion}

The 3DR-MC algorithm was adopted to compare the effects of the T-MC algorithm and the MS-MC algorithm in reconstructing the left and right lung lobes. It was found that the algorithm in this study had a shorter running time on the platform in contrast to that of the other algorithms. After it was applied in the CTPA of PE patients, it was found that $\mathrm{PE}+\mathrm{S}$ was the result of multifactor interaction, and the incidence of syncope in PE patients was not low, and the mortality rate could be increased, so it required a high degree of vigilance. The deficiency of this study is that the risk factors considered in the diagnostic criteria of syncope are not comprehensive enough, which will have an impact on the results of the study, more comprehensive risk factors need to be included in the later stage to further explore. All in all, $\mathrm{PE}+\mathrm{S}$ patients had certain clinical characteristics, based on which the disease could be diagnosed as early as possible. It was helpful for correct treatment and reducing the adverse consequences caused by improper diagnosis and treatment.

\section{Data Availability}

The data used to support the findings of this study are available from the corresponding author upon request.

\section{Conflicts of Interest}

The authors declare no conflicts of interest.

\section{Authors' Contributions}

Zhongxing Zhang and Yan Zhang contributed equally to this work.

\section{Acknowledgments}

This work was supported by the Chongqing Science and Technology Bureau project (nos. 2016jstg006, 2017jstg31, and 2019jstg023).

\section{References}

[1] V. Palm, F. Rengier, P. Rajiah, C. P. Heussel, and S. Partovi, "Acute pulmonary embolism: imaging techniques, findings, endovascular treatment and differential diagnoses," Röfo: Fortschritte auf dem Gebiete der Rontgenstrahlen und der Nuklearmedizin, vol. 192, no. 1, pp. 38-49, 2020.

[2] M. Hu, Y. Zhong, S. Xie, H. Lv, and Z. Lv, "Fuzzy system based medical image processing for brain disease prediction," Frontiers in Neuroscience, vol. 15, Article ID 714318, 2021.

[3] V. Thiruganasambandamoorthy, M. L. A. Sivilotti, B. H. Rowe et al., "Prevalence of pulmonary embolism among emergency department patients with syncope: a multicenter prospective cohort study," Annals of Emergency Medicine, vol. 73, no. 5, pp. 500-510, 2019.

[4] A. Parakh, F. Macri, and D. Sahani, "Dual-energy computed tomography," Radiologic Clinics of North America, vol. 56, no. 4, pp. 601-624, 2018.

[5] Z. Wan, Y. Dong, Z. Yu, H. Lv, and Z. Lv, "Semi-supervised support vector machine for digital twins based brain image fusion," Frontiers in Neuroscience, vol. 15, Article ID 705323, 2021.

[6] G. Chacón, J. E. Rodríguez, V. Bermúdez et al., "Computational assessment of stomach tumor volume from multi-slice computerized tomography images in presence of type 2 cancer," F1000Research, vol. 7, 2018.

[7] Task Force for the Diagnosis and Management of Syncope, European Society of Cardiology (ESC), European Heart Rhythm Association (EHRA) et al. "Guidelines for the diagnosis and management of syncope (version 2009)," European Heart Journal, vol. 30, no. 21, pp. 2631-2671, 2009.

[8] S. V. Konstantinides, "2014 ESC Guidelines on the diagnosis and management of acute pulmonary embolism," European Heart Journal, vol. 35, no. 45, pp. 3145-3146, 2014.

[9] M. R. Jaff, M. S. McMurtry, S. L. Archer et al., "Management of massive and submassive pulmonary embolism, iliofemoral deep vein thrombosis, and chronic thromboembolic 
pulmonary hypertension," Circulation, vol. 123 , no. 16, pp. 1788-1830, 2011.

[10] M. Cecconi, D. De Backer, M. Antonelli et al., "Consensus on circulatory shock and hemodynamic monitoring. Task force of the European Society of Intensive Care Medicine," Intensive Care Medicine, vol. 40, no. 12, pp. 1795-1815, 2014.

[11] B. Rivera-Lebron, M. McDaniel, K. Ahrar et al., "PERT consortium. diagnosis, treatment and follow up of acute pulmonary embolism: consensus practice from the pert consortium," Clinical and Applied Thrombosis/Hemostasis, vol. 25, Article ID 1076029619853037, 2019.

[12] D. Epstein, G. Berger, N. Barda et al., "The incidence of acute pulmonary embolism following syncope in anticoagulantnaïve patients: a retrospective cohort study," PLoS One, vol. 13, no. 3, Article ID e0193725, 2018.

[13] C. P. Monté, C. J. Monté, P. Boon, and J. Arends, "Epileptic seizures associated with syncope: ictal bradycardia and ictal asystole," Epilepsy and Behavior: E\&B, vol. 90, pp. 168-171, 2019.

[14] K. M. Sink, G. W. Evans, R. I. Shorr et al., "Syncope, Hypotension, and falls in the treatment of hypertension: results from the randomized clinical systolic blood pressure intervention trial," Journal of the American Geriatrics Society, vol. 66, no. 4, pp. 679-686, 2018.

[15] M. V. Di Maria, K. R. Campbell, D. A. Burkett et al., "Parameters of right ventricular function reveal ventricularvascular mismatch as determined by right ventricular stroke work versus pulmonary vascular resistance in children with pulmonary hypertension," Journal of the American Society of Echocardiography, vol. 33, no. 2, pp. 218-225, 2020.

[16] S. Xie, Z. Yu, and Z. Lv, "Multi-disease prediction based on deep learning: a survey," Computer Modeling in Engineering and Sciences, vol. 127, no. 3, pp. 1-34, 2021.

[17] H. İdil and T. Y. Kilıc, "Diagnostic yield of neuroimaging in syncope patients without high-risk symptoms indicating neurological syncope," The American Journal of Emergency Medicine, vol. 37, no. 2, pp. 228-230, 2019.

[18] A. E. Menyar, K. Sulaiman, A. AlSadawi et al., "Implications of a history of syncope in patients hospitalized with heart failure," Angiology, vol. 68, no. 3, pp. 196-206, 2017. 